\title{
SIMULAÇÃO DINÂMICA DE UMA REDE DE TROCADORES DE CALOR
}

\author{
E. L. R. TONASSI, J. C. LEMOS, E. R. A. LIMA e A. L. H. COSTA
}

Universidade do Estado do Rio de Janeiro, Programa de Pós Graduação em Engenharia Química

E-mail para contato: andrehc@uerj.br

\begin{abstract}
RESUMO - Redes de Trocadores de calor são estruturas amplamente utilizadas na indústria petroquímica, sendo formadas por um conjunto de trocadores que promovem a transferência de calor entre as correntes quentes e frias. A simulação dinâmica destas estruturas é muito importante para prever seu comportamento e sua resposta a possíveis perturbações. O presente trabalho apresenta a simulação dinâmica destes sistemas. O modelo é composto por um conjunto de equações diferenciais de balanço de massa e energia, complementadas por equações algébricas associadas à interconexão entre os equipamentos, sendo capaz de representar redes com qualquer padrão, incluindo reciclos, by-passes, loops, etc. Numericamente, o sistema resultante é discretizado no espaço via diferenças finitas e integrado no tempo utilizando o código DASSL (métodos das linhas). A aplicação da ferramenta de simulação desenvolvida é ilustrada através de um exemplo de redes de trocadores de calor de caráter industrial.
\end{abstract}

\section{INTRODUÇÃO}

A troca de calor entre fluidos de diferentes temperaturas é de suma importância e possui diversas aplicações na engenharia. Na indústria de processos químicos, trocadores de calor são responsáveis pela alteração da temperatura e/ou estado físico de correntes materiais. Visando diminuir o consumo de energia, sempre que possível, correntes do próprio processo são utilizadas para resfriar ou aquecer outras, formando uma rede de troca térmica integrada.

Apesar de estas estruturas serem capazes de reduzir consideravelmente o consumo de utilidades, implicam em sistemas de maior complexidade do ponto de vista operacional. Dessa forma, ferramentas de simulação especializadas assumem uma maior importância como suporte à engenharia de processos na análise desses sistemas.

Devido à sua importância em um cenário de aumento dos custos de energias, a literatura contém um grande número de trabalhos focados na síntese de redes de trocadores de calor (Furman e Sahinidis, 2002). Entretanto, os trabalhos devotados às ferramentas de simulação são bem mais limitados (Rodera et al., 2003) e, basicamente, voltados à simulação em regime permanente (Ratnam e Patwardhan, 1991; Wang e Sundén, 2001; Picón-Núñez et al., 2002; Oliveira Filho et al., 2007).

Neste contexto, o presente trabalho apresenta uma ferramenta de simulação capaz de descrever 


\section{9 a 22 de outubro de 2014 \\ Florianópolis/SC}

o comportamento dinâmico de redes de trocadores de calor sujeitas à perturbação térmica. $\mathrm{O}$ modelo matemático da rede é composto por um conjunto de balanços de massa e energia em cada componente da rede. A conectividade entre os diversos elementos da rede é descrita através da representação da rede na forma de um dígrafo, parametrizada na forma de uma matriz de incidência. Esta organização permite simular redes com qualquer padrão de interconexão, incluindo reciclos, by-passes, loops, etc.

Do ponto de vista matemático, o modelo empregado na simulação corresponde a um sistema de equações algébrico-diferenciais. Numericamente, as equações diferenciais parciais são discretizadas no espaço via diferenças finitas e integradas no tempo (método das linhas), juntamente com as equações algébricas utilizando o código DASSL.

\section{MODELAGEM DOS EQUIPAMENTOS}

As redes de trocadores de calor investigadas são compostas pelos seguintes elementos: trocadores de calor, misturadores, divisores de correntes, pontos de suprimento e pontos de demanda. Os pontos de suprimento e demanda correspondem à representação dos equipamentos externos à rede.

\subsection{Trocadores de Calor}

No modelo apresentado, são considerados trocadores de calor casco-e-tubo com um passe no casco e $\mathrm{N}$ passes nos tubos, sendo $\mathrm{N}$ um número par. Esta alternativa de trocador é muito comum em redes industriais. Esta alternativa de trocador é muito comum em redes industriais, entretanto, o modelo proposto também pode ser diretamente adaptado para outras configurações, tais como contracorrente ou cocorrente. Da mesma forma, devido à limitação do espaço disponível, será considerado apenas o escoamento da corrente fria no lado dos tubos e da corrente quente no lado do casco, mas tal orientação pode ser facilmente estendida para a alocação inversa. De acordo com estas orientações, as hipóteses adotadas no modelo são: variações da energia cinética e potencial desprezíveis, propriedades físicas do fluido constantes, balanço de energia unidimensional, difusão axial desprezível, e coeficiente global de troca térmica constante.

De acordo com as premissas estabelecidas, o balanço de energia corresponde a uma equação para a corrente quente e $N$ equações para a corrente fria, uma para cada passe, identificados pelo índice $j=1, \ldots, N$ :

$$
\begin{aligned}
& \frac{\partial \theta_{c, j}}{\partial t}=(-1)^{j} \frac{m_{c}}{\rho_{c} A_{e s c t}} \frac{\partial \theta_{c, j}}{\partial z}+\frac{U P\left(\theta_{h}-\theta_{c, j}\right)}{\rho_{c} A_{e s c t} C p_{c} N p t} \\
& \frac{\partial \theta_{h}}{\partial t}=\frac{m_{h}}{\rho_{h} A_{e s c s}} \frac{\partial \theta_{h}}{\partial z}+\frac{U P\left(N \theta_{h}-\sum \theta_{c, j}\right)}{\rho_{c} A_{e s c s} C p_{h}}
\end{aligned}
$$

onde os subscritos $c$ e $h$ representam as correntes frias e quente; $\theta$ corresponde à temperatura das correntes ao longo do comprimento do trocador; $t$ é o tempo de simulação; $z$ é o comprimento do trocador; $m, \rho$ e $C p$ são a vazão mássica, a massa específica e a capacidade calorífica das correntes; $P$ 
é o perímetro da área de troca térmica; Npt é o número de passes nos tubos; $A_{\text {esct }}$ é a área de escoamento nos tubos e $A_{\text {escs }}$ é a área de escoamento no casco.

As Equações (1) e (2) são discretizadas via diferenças finitas centrais, resultando nas seguintes equações diferenciais ordinárias, nas quais cada ponto da malha é identificado pelo índice $i$ :

$$
\begin{aligned}
& \frac{d \theta_{c, j}^{i}}{d t}=(-1)^{j} \frac{m_{c}}{\rho_{c} A_{e s c t}} \frac{\theta_{c, j}^{i+1}-\theta_{c, j}^{i-1}}{2 h}+\frac{U P\left(\theta_{h}^{i}-\theta_{c, j}^{i}\right)}{\rho_{c} A_{e s c t} C p_{c} N p t} \\
& \frac{d \theta_{h}^{j}}{d t}=\frac{m_{h}}{\rho_{h} A_{e s c s}} \frac{\theta_{h}^{i+1}-\theta_{h}^{i-1}}{2 h}+\frac{U P\left(N \theta_{h}^{i}-\sum \theta_{c, j}^{i}\right)}{\rho_{c} A_{e s c s} C p_{h}}
\end{aligned}
$$

onde $h$ corresponde ao intervalo entre os pontos na malha.

\subsection{Misturador de Correntes}

Um misturador de correntes é considerado como um ponto de mistura que recebe duas correntes do processo e as une em uma única corrente na saída do equipamento. Como a dinâmica deste processo é considerada desprezível, têm-se para os balanços de energia a seguinte equação algébrica:

$$
C_{i, 1} T_{i, 1}+C_{i, 2} T_{i, 2}+C_{i, 3} T_{i, 3}+\ldots-C_{o} T_{o}=0
$$

onde os subscritos $i$ e $o$ representam a entrada e saída das correntes no equipamento, $C$ é a taxa de capacidade calorífica da corrente (produto da vazão mássica pela capacidade calorífica) e $T$ é a temperatura das correntes.

\subsection{Divisor de Correntes}

Um divisor de correntes corresponde a um ponto onde uma corrente do processo é dividida em duas correntes de saída. De acordo com a mesma hipótese adotada para o misturador, o balanço de energia assume a seguinte forma:

$$
\begin{aligned}
& C_{i} T_{i}-C_{o, 1} T_{o, 1}-C_{o, 2} T_{o, 2}=0 \\
& T_{i}-T_{o, 1}=0
\end{aligned}
$$

\subsection{Pontos de Suprimento e Demanda}

Os pontos de suprimento e demanda representam a entrada e a saída das correntes na rede. O balanço de energia para os pontos de suprimento (entrada de fluido na rede oriundo de um equipamento externo) e demanda (saída de fluido da rede para um equipamento externo) podem ser representados por: 


$$
\begin{aligned}
& V^{P S}+T_{o}=0 \\
& V^{P S}+T_{i}=0
\end{aligned}
$$

onde $V$ é a temperatura correspondente a corrente externa e os sobrescritos $P S$ e $P D$ se aplicam aos pontos de suprimento e de demanda, respectivamente.

\section{MODELAGEMA DA REDE}

A modelagem da rede é composta pelo encadeamento dos modelos dos equipamentos de acordo com a interconexão destes através das correntes. A rede é descrita na forma de um dígrafo onde os vértices representam os equipamentos e as arestas as correntes de interligação. Através da parametrização da estrutura da rede na forma de uma matriz de incidência, o modelo assume uma forma matricial. Neste contexto, a matriz de incidência da rede é particionada da seguinte forma de acordo com os elementos da rede e das correntes quentes e frias (Oliveira Filho et al., 2007):

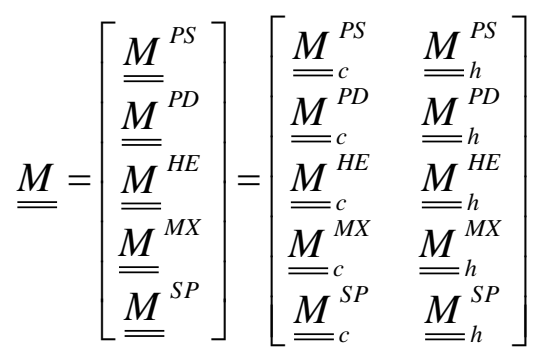

onde os sobrescritos $P S, P D, H E, M X$ e $S P$ representam os nós de suprimento, demanda, trocadores de calor, misturadores, e divisores de corrente, respectivamente.

\subsection{Trocadores de Calor}

A representação matricial das Equações (3) e (4) é dada por:

$$
\frac{d \underline{\theta}}{d t}=\underline{\underline{\Lambda}} \underline{\theta}
$$

onde $\underline{\theta}$ corresponde ao vetor de temperaturas ao longo dos diversos trocadores de calor e $\underline{\underline{\Lambda}}$ corresponde à matriz de coeficientes. Esta matriz contém submatrizes tridiagonais, oriundas dos coeficientes associados às correntes frias e submatrizes identidade, oriundas dos coeficientes associados às correntes quentes.

A interconexão entre as correntes quentes e frias no interior dos trocadores com as correntes quentes e frias da rede (i.e. que interconectam os equipamentos) são dadas por:

$$
\underline{\Gamma}_{c, i} \underline{\theta}=\left[\left(\underline{\underline{M}}_{c}^{H E}\right)_{+}\right] \underline{T}_{c}
$$




$$
\begin{aligned}
& \underline{\Gamma}_{h, i} \underline{\theta}=\left[\left(\underline{\underline{M}}_{h}^{H E}\right)_{+}\right] \underline{T}_{h} \\
& \underline{\Gamma}_{c, o} \underline{\theta}=\left[\left(\underline{\underline{M}}_{c}^{H E}\right)_{-}\right] \underline{T}_{c} \\
& \underline{\Gamma}_{h, o} \underline{\theta}=\left[\left(\underline{\underline{M}}_{h}^{H E}\right)_{+}\right] \underline{T}_{h}
\end{aligned}
$$

onde cada linha das matrizes $\underline{\underline{\Gamma}}$ corresponde a um dos trocadores de tal forma que a coluna correspondente à corrente fria/quente de entrada/saída é igual a 1 sendo as demais iguais a zero.

\subsection{Misturador e Divisor de Correntes}

A representação matricial das Equações (5), (6) e (7) no contexto da rede completa assume a seguinte forma:

$$
\begin{aligned}
& \underline{\underline{M}}^{M X}[\underline{\underline{\operatorname{diag}}}(\underline{C})] \underline{T}=\underline{0} \\
& \left.\underline{\underline{M}}^{S P}[\underline{\underline{\operatorname{diag}}} \underline{\underline{C}})\right] \underline{T}=\underline{0} \\
& {\left[\underline{\underline{M}}_{+}^{S P}-\underline{\underline{S P}}\right] \underline{T}=\underline{0}}
\end{aligned}
$$

onde o subscrito + indica a eliminação dos elementos negativos da matriz correspondente. Cada linha da matriz $\underline{S P}$ corresponde a um divisor de corrente de tal forma que a coluna correspondente a uma das correntes de saída tem valor igual a 1 e as demais igual a zero.

\subsection{Pontos de Suprimento e Demanda}

As Equações (8) e (9) são representadas na rede por:

$$
\begin{aligned}
& \underline{\underline{M}}^{P S} \underline{T}+\underline{V}^{P S}=\underline{0} \\
& \underline{\underline{M}}^{P D} \underline{T}-\underline{V}^{P D}=\underline{0}
\end{aligned}
$$

\subsection{Especificações}

As especificações da rede correspondem às temperaturas das correntes de entrada oriundas dos pontos de suprimento são descritas por:

$$
\underline{V}^{P S}-\left(\underline{V}^{P S}\right)^{*}=\underline{0}
$$


onde o sobrescrito * indica os valores das especificações

\section{SIMULAÇÃO DA REDE}

O modelo da rede a ser resolvido na simulação é composto pelo sistema de equações diferenciais lineares de primeira ordem apresentado na Equação (11) e pelo sistema de equações algébricas lineares representado pelas Equações (12) a (21).

Este sistema é resolvido por meio da rotina DASSL disponível no software Scilab.

\section{RESULTADOS}

\subsection{Exemplo Ilustrativo}

O desempenho do modelo proposto é ilustrado por meio da simulação de uma perturbação degrau em uma rede de seis trocadores de calor inicialmente em regime permanente. Esses trocadores são organizados em dois ramais em paralelo de três trocadores, tal como ilustra a Figura 1. A estrutura desta rede foi empregada originalmente na investigação de paradas para limpeza em Assis et al. (2013).

Todos os trocadores de calor presentes na rede são iguais, apresentando uma configuração do tipo casco-e-tubo com 2 passes nos tubos e 1 passe no casco, e área de troca térmica igual a $400 \mathrm{~m}^{2}$. Os tubos dos trocadores de calor possuem diâmetro interno e externo iguais a 14,85 mm e 19,05 mm, com comprimento de 4,46 m. O coeficiente global de troca térmica foi considerado constante e igual a $857 \mathrm{~W} / \mathrm{m}^{2} \mathrm{~K}$. As especificações das correntes de entrada associadas ao regime estacionário são também apresentadas na Figura 1, em que a divisão das correntes entre os diferentes ramais e trocadores é igual.

De forma preliminar, o sistema de equações do modelo foi resolvido impondo valores nulos para as derivadas, de maneira a identificar a condição operacional de regime permanente. Os valores encontrados foram idênticos àqueles originalmente reportados em Assis et al. (2013).

A partir da condição de estado estacionário, o algoritmo de simulação foi aplicado a um cenário no qual a temperatura da corrente fria que alimenta a rede sofre uma variação de $40{ }^{\circ} \mathrm{C}$. A resposta dinâmica das correntes de cada ramal é apresentada na Figura 2. 


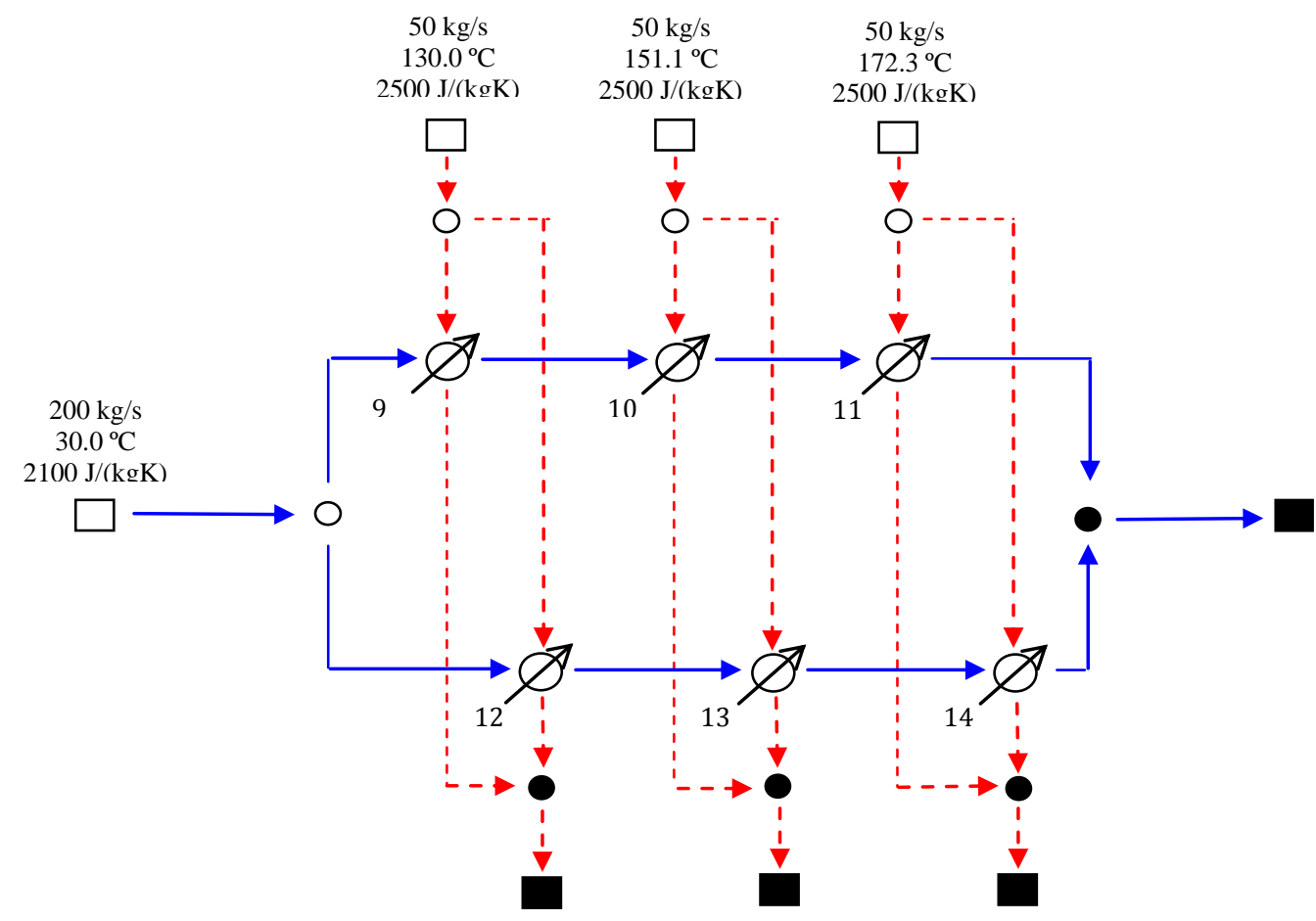

Figura 1 - Rede de trocadores de calor investigada

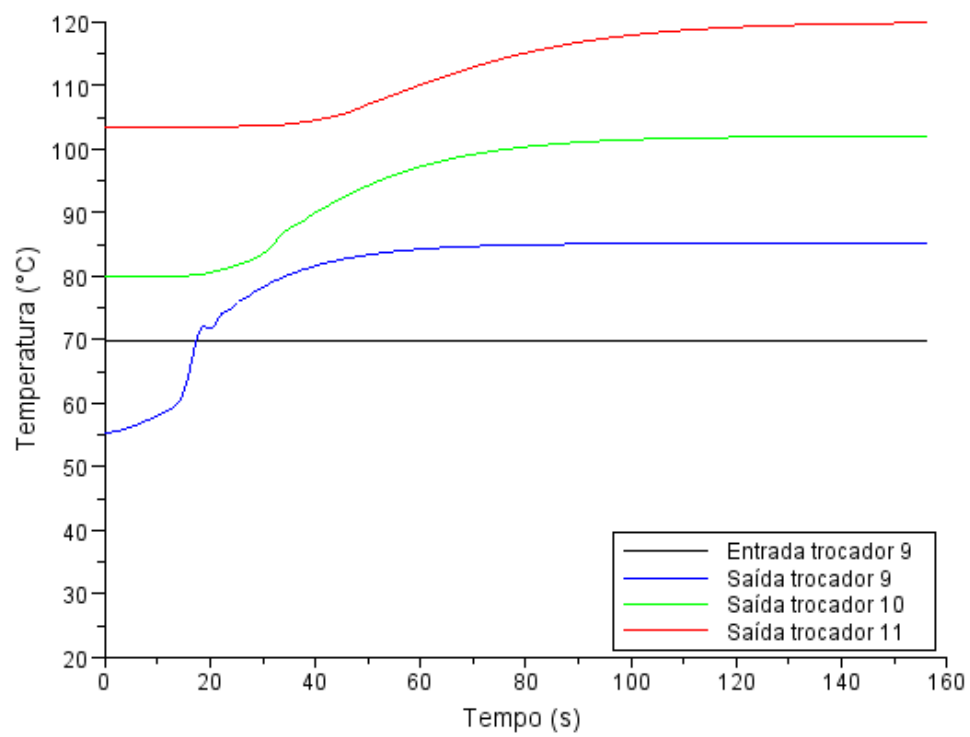

Figura 2 - Perfil das temperaturas após perturbação 
Ao realizar a perturbação na temperatura do fluido frio de entrada da rede, entrada do trocador 9, um novo estado estacionário será alcançado, como pode ser observado na Figura 2.

A temperatura de saída do trocador 9 atinge esse novo estado antes da temperatura de saída do trocador 10, que atinge antes da temperatura de saída do trocador 11. Este comportamento era esperado, pois os trocadores mais próximos da entrada demoram menos tempo para responder à variação, ocorrendo o oposto para os mais distantes. Essa análise foi feita apenas para um dos ramais da corrente fria, já que o comportamento do outro é idêntico.

\section{CONCLUSÕES}

O presente trabalho apresentou um modelo dinâmico de redes de trocadores de calor representado totalmente de forma matricial. A abordagem proposta é bastante flexível podendo acomodar qualquer estrutura de rede na forma de um sistema algébrico-diferencial linear.

Através de um exemplo de rede de trocadores de calor, a utilização da ferramenta de simulação é ilustrada, de tal forma que a perturbação em uma temperatura de entrada é propagada ao longo dos trocadores existentes nos ramais.

\section{REFERÊECIAS}

ASSIS, B. C. G., LEMOS, J. C., QUEIROZ, E. M., PESSOA, F. L. P., LIPORACE, F. S., OLIVEIRA, S. G., COSTA, A. L. H., Optimal allocation of cleanings in heat exchanger networks. App. Therm. Eng., v. 58, p. 605-614, 2013.

FURMAN, K. C., SAHINIDIS, N. V. A critical review and annotated bibliography for heat exchanger network synthesis in the 20th century, Ind. Eng. Chem. Res., v. 41, p. 2335-2370, 2002.

OLIVEIRA FILHO, L. O., QUEIROZ, E. M., COSTA, A. L. H. A matrix approach for steady-state simulation of heat exchanger networks. App. Therm. Eng., v. 27, p. 2385-2393, 2007.

PICÓN-NÚÑEZ, M., CASTRO-PÁEZ, J., VIZCAÍNO-GARCÍA, F., Steady state simulation for the de-bottlenecking of heat recovery networks, App. Therm. Eng., v. 22, p. 1673-1687, 2002.

RATNAM, R., PATWARDHAN, V.S., Sensitivity analysis for heat exchanger networks, Chem. Eng. Sci., v. 46, p. 451-458, 1991.

RODERA, H., WESTPHALEN, D. L., SHETHNA, H. K., A methodology for improving heat exchanger network operation, App. Therm. Eng., v. 23, p. 1729-1741, 2003.

WANG, L., SUNDÉN, B., Detailed simulation of heat exchanger networks for flexibility consideration, App. Therm. Eng., v. 21, p. 1175-1184, 2001. 